\section{Omega-3 fatty acid ethyl esters do not improve clopidogrel associated P2Y12 inhibition in stroke patients}

\author{
Ping Li, ${ }^{1}$ Haris Kamal, ${ }^{1}$ Melissa Baxter,2 \\ Bijal K. Mehta ${ }^{3}$ \\ 1Department of Neurology, Jacob \\ Neurological Institute, University at \\ Buffalo, NY; 2Department of Pharmacy, \\ Buffalo General Medical Center, \\ University at Buffalo, NY; ${ }^{3}$ Department \\ of Neurology, David Geffen School of \\ Medicine, University of California Los \\ Angeles, Harbor-UCLA Medical Center, \\ Torrance, CA, USA
}

\section{Abstract}

The specific action of omega- 3 fatty acid ethyl esters (OFA) in preventing cerebrovascular disease remains unknown, but research has demonstrated multiple possible mechanisms. In addition to altering lipid profiles, OFA may inhibit platelet aggregation. Clopidogrel inhibits platelets via the P2Y12 receptor. OFA may alter clopidogrel-associated platelet-inhibition via a possible combined effect on P2Y12 inhibition. To determine if OFA affects clopidogrel associated P2Y12 platelet receptor inhibition by comparing the percentage of responders in patients with cerebrovascular disease who were taking clopidogrel with or without OFA. We retrospectively reviewed data from adult patients with cerebrovascular disease or cerebral aneurysms and taking clopidogrel, who were seen at a single hospital between March 2010 to September 2011. We included 438 subjects in the study. For the 67 subjects who received loading doses of both clopidogrel and OFA, $71.6 \%$ had a P2Y12 inhibition response more than $20 \%$, which is considered a positive response. For the 55 subjects who received just clopidogrel load, $67.2 \%$ of subjects were responders. There were $70.4 \%$ responders in the 274 subjects who were taking $75 \mathrm{mg}$ of clopidogrel alone at home, and $73.8 \%$ responders in the 42 subjects who were taking both clopidogrel and OFA at home. However, these percentage differences were not statistically significant. This study did not find additional P2Y12 platelet inhibition when patients were given OFA, either given as a loading dose or taking it daily.

\section{Introduction}

Several trials have confirmed that antiplatelet agents like aspirin, clopidogrel, and aspirin-dipyridamole do provide secondary stroke prevention.1,2 Therefore, the use of antiplatelet agents is a mainstay of stroke management. ${ }^{3}$ Although the readily available platelet function tests have made the determination of patients' responses to certain antiplatelets more objective, their use in management of stroke prevention remains a continued area of research.

Additionally, the use of other agents for secondary stroke prevention such as HMG-CoA inhibitors is also considered a valid addition to a post-stroke prevention regime.3,4 In addition to these medications, other over the counter and complementary medicines have been investigated concurrently with these medications. One such class of complementary medicines includes omega-3 fatty acid ethyl esters (OFA), such as docosahexaenoic acid (DHA) and eicosapentaenoic acid (EPA).

Omega-3 fatty acids are a class of fatty acids of essential nutrients, since they cannot be synthesized by humans and must be obtained in the diet. However, most western diets are rich in omega- 6 fatty acids and not largely fish derived omage- 3 fatty acids. The hypothesized result is that low omega- 3 fatty acid diets result in high rates of poor serum lipid profiles and subsequent vascular disease, including stroke and coronary artery disease. ${ }^{5-7}$

The mechanism of how these fats improve serum lipid proportions and how they contribute to a less inflammatory state continues to be elucidated. One area where omega- 3 fatty acids have an effect appears to be platelet aggregation. 0mega-3 fatty acids appear to compete with omega- 6 fatty acid enzymes that convert omega- 3 fatty acids into category 3 thromboxanes and prostaglandins instead of category 1 and 2 thromboxanes and prostaglangins from omega- 6 fatty acids. With less thromboxane $\mathrm{A} 2$ and more $\mathrm{A} 3$, this ultimately leads to less glycoprotein IIb/IIIa signaling resulting less in platelet activation and binding to fibrinogen. ${ }^{5}$ Also, the P2Y12 receptor on platelets is another mechanism that can amplify the glycoprotein IIb/IIIa integrin signaling. Antiplatelet agents such as aspirin and clopidogrel inhibit the cox-2 enzyme and P2Y12 receptor, respectively, which decreases platelet-platelet aggregation. ${ }^{8}$

Data in the cardiac literature has shown that there was lower rate of clopidogrel resistance (non-responders) after taking OFA with clopidogrel and aspirin for 30 days compared to those taking clopidogrel and aspirin without OFA. ${ }^{9}$ Although it is not a common practice, it has been extrapolated to give OFA to clopidogrel non-responders with cerebrovascular dis-
Correspondence: Bijal K. Mehta, Department of Neurology, David Geffen School of Medicine, University of California Los Angeles, HarborUCLA Medical Center, 1000 West Carson Street Torrance, CA 90509, USA

Tel.: + 1.310.222.3897.

E-mail: bijal@yahoo.com

Key words: Omega-3 fatty acid; clopidogrel; P2Y12 receptor; cerebrovascular disease; stroke; DHA; EPA.

Contributions: the authors contributed equally.

Conflict of interest: the authors declare no potential conflict of interest.

Received for publication: 13 January 2015. Accepted for publication: 17 April 2015.

This work is licensed under a Creative Commons Attribution NonCommercial 3.0 License (CC BYNC 3.0).

(C) Copyright P. Li et al., 2015

Licensee PAGEPress, Italy

Neurology International 2015; 7:5809

doi:10.4081/ni.2015.5809

ease. The purpose of this study is to determine if OFA use in patients with cerebrovascular disease can improve clopidogrel associated P2Y12 platelet inhibition by comparing: i) the percentage of responders in cerebrovascular disease patients given clopidogrel load along with OFA and patients only receiving loading doses of clopidogrel; ii) the percentage of responders in cerebrovascular disease patients who were taking both a standard dose $75 \mathrm{mg}$ daily) of clopidogrel and OFA with patients only taking the standard dose of clopidogrel at home.

\section{Materials and Methods}

This is a retrospective chart review study of patients (19 to 98 years old) with cerebrovascular disease or cerebral aneurysms who received clopidogrel in a single high volume stroke center. The study was approved by Health Science IRB of the University at Buffalo. These patients were seen consecutively between March 2010 and September 2011. Only patients who were on clopidogrel prior to arrival to the hospital or started at the hospital for cerebrovascular disease were included in the study. Patients who were taking clopidogrel only for cardiac reasons, patients whose P2Y12 platelet inhibition tests were done prior to the administration of clopidogrel, and those who did not have P2Y12 platelet inhibition test were excluded. 
Additionally, patients on proton pump inhibitor medications (known competitive inhibitor of clopidogrel metabolism), 10 and those who did not have a mediation list on record were excluded. Whether patients were on omega-3 supplementation (either prescription or OverThe-Counter) prior to arrival to the hospital or started in combination with clopidogrel were recorded. From the medical records, demographic information, the dosages of clopidogrel and OFA, and patients' significant past medical histories were recorded.

The VerifyNow P2Y12 point-of-care assay (Accumetrics, San Diego, CA, USA) was used to measure platelet response. VerifyNow P2Y12 is a rapid platelet function assay designed to measure directly the effects of drugs on the P2Y12 receptor. Results of $20 \%$ or greater inhibition ( $\mathrm{PRN}<237$ ) was considered as adequate response of platelet inhibition. ${ }^{11}$ The percentage inhibition was based on P2Y12 Reaction Units (PRU) and Base PRU, with Base as maximal platelet aggregation via thrombin receptor activating peptide pathway.

Statistical analysis was performed using Chi-square test for the categorical variables and student's t-test for the continuous variables. Multiple logistic regression model was used to investigate the effects of OFA on P2Y12 inhibition controlling hypertension (HTN), coronary artery disease (CAD), and dyslipidemia. Significance was defined as $\mathrm{P}<0.05$. All statistics were performed by using SAS version 9.1 (SAS Inc, Chicago, IL, USA).

\section{Results}

Among 2681 consecutive patients who were given clopidogrel during the study period, 438 patients were included in the study. Among the patients who were excluded, 1808 patients did not have clopidogrel platelet inhibition test, 15 did not receive any clopidogrel before the P2Y12 inhibition test, 109 did not have a detailed medication list on record, 268 were on clopidogrel for cardiac reasons only, and 43 were taking proton pump inhibitor. The included subjects were taking clopidogrel for either secondary stroke prevention $(203,46.3 \%)$ or stent placement in carotid or vertebral-basilar arteries (235, 53.7\%).

In the 122 patients who received loading doses of clopidogrel, 67 (54.9\%) received both loading doses of clopidogrel and OFA, and 55 (45.1\%) received only clopidogrel loading dose. The patients in the two groups were around the same age $(66.6 \pm 12.7$ versus $67.8 \pm 12.9$, respectively; Table 1 ). There were no significant differences in the responder rates either with OFA or without ( $71.6 \%$ versus $67.2 \%)$, or in the mean percentage platelet inhibitions $(29.1 \%$ versus $32.5 \%)$. The frequency of patients with hypertension, dyslipidemia, diabetes mellitus, coronary artery disease, taking aspirin concurrently, and current smoking status did not differ between the two groups as well (Table 1). The loading doses of clopidogrel in the prior 24-hour and 48-hour of P2Y12 platelet inhibition test were comparable between the two groups $(619.8 \pm 358.4 \mathrm{mg}$ versus $568.6 \pm 347.2 \mathrm{mg}$ for 24-hour, and $901.2 \pm 443.5 \mathrm{mg}$ versus $785.5 \pm 342.1 \mathrm{mg}$ for 48 hour).

In the 316 patients who were taking daily dose of clopidogrel (75 mg per day) at home, 42 (13.3\%) were taking OFA daily (ranging from $300 \mathrm{mg}$ to $4000 \mathrm{mg}$ per day) as well. The age and gender between the two groups were similar (Table 2). Although the responder rate was higher in patients who were taking both clopidogrel and OFA at home (73.8\%) than in patients who were not $(70.4 \%)$, there was no significant difference after controlling for different variables (hypertension, dyslipidemia, and coronary artery disease) using multiple logistic regression model $(\mathrm{P}=0.522)$. The mean percentage of platelet inhibitions between the two groups were also not signifi-

Table 1. The characteristics of subjects who received clopidogrel loads.

\begin{tabular}{lcc} 
& With OFA & Without OFA \\
Number & $67(54.9 \%)$ & $55(45.1 \%)$ \\
Age & $66.6 \pm 12.7$ & $67.8 \pm 12.9$ \\
\hline Male & $40(59.7 \%)$ & $31(56.4 \%)$ \\
Percentage inhibition & $29.9 \pm 19.7$ & $32.5 \pm 24.6$ \\
\hline Responders & $47(70.1 \%)$ & $37(67.2 \%)$ \\
Hypertension & $55(82.1 \%)$ & $46(83.6 \%)$ \\
\hline Dyslipidemia & $52(77.6 \%)$ & $46(83.6 \%)$ \\
Diabetes mellitus & $30(44.8 \%)$ & $19(34.5 \%)$ \\
\hline Coronary artery disease & $30(44.8 \%)$ & $24(43.6 \%)$ \\
On aspirin concomitantly & $62(92.5 \%)$ & $50(90.9 \%)$ \\
\hline Current smoker & $16(23.9 \%)$ & $21(38.2 \%)$ \\
\hline
\end{tabular}

OFA, omega-3 fatty acid ethyl esters.

Table 2. The characteristics of subjects who were taking clopidogrel at home.

\begin{tabular}{lcc} 
& With OFA & Without OFA \\
Number & $42(13.3 \%)$ & $274(86.7 \%)$ \\
\hline Mge & $72.1 \pm 12.5$ & $69.5 \pm 13.6$ \\
Male & $19(45.2 \%)$ & $156(56.9 \%)$ \\
Percentage inhibition & $41.6 \pm 28.8$ & $38.9 \pm 27.6$ \\
Hypertension & $31(73.8 \%)$ & $193(70.4 \%)$ \\
Dyslipidemia & $40(95.2 \%)$ & $224(81.8 \%)^{*}$ \\
Diabetes mellitus & $34(80.9 \%)$ & $187(68.2 \%)$ \\
Coronary artery disease & $10(23.8 \%)$ & $89(32.5 \%)$ \\
On aspirin concomitantly & $26(61.9 \%)$ & $107(39.1 \%)^{*}$ \\
Current smoker & $38(90.5 \%)$ & $230(83.9 \%)$ \\
\hline
\end{tabular}

OFA, omega-3 fatty acid ethyl esters. ${ }^{*} \mathrm{P}<0.05$. cant $(41.6 \pm 28.8$ for those who were taking both clopidogrel and OFA at home vs. $38.9 \pm 27.6$ for those who were taking clopidogrel but not OFA at home). There were more subjects who were on both medications at home having hypertension or coronary artery disease, which were likely the reasons for them to take OFA chronically (Table 2). The frequency of patients with dyslipidemia, diabetes mellitus, on concurrent aspirin, and current smoking status did not differ between the two groups.

\section{Discussion}

The use of OFA in patients with vascular diseases remains controversial. However, it is prescribed in patients with vascular risk factors including dyslipidemia, coronary artery disease, and ischemic stroke with some evidence showing beneficial results.9,12-15 Our study demonstrates that OFA do not appear to impair platelet function via the P2Y12 receptor; therefore taking OFA cannot lower the rate 
of clopidogrel resistance. Gajos et al. reported that the addition of OFA to the combination of aspirin and clopidogrel significantly potentiates platelet response to clopidogrel after percutaneous coronary intervention. ${ }^{9}$ However, in this study $5 \mathrm{~mol} / \mathrm{L}$ ADP was used as the agonist when determining the clopidogrel platelet inhibition, which had been shown having higher coefficients of variation for light transmission aggregation comparing to $20 \mathrm{~mol} / \mathrm{L}$ ADP as used in VerifyNow assay P2Y12 assay. 16 The definition of low responders to clopidogrel was also different. The difference of the methodology between the studies besides different patient populations might explain the difference in the results. One prior cardiac study was able to show ADP and glycoprotein IIb/IIIa inhibition with omega-3 fatty acid supplementation, but like our study in stroke patients, it was not able to show a statistically significant increase in platelet inhibition using the VerifyNow assay test. ${ }^{17}$ The VerifyNow P2Y12 point-of-care assay calculates inhibition using measurements of light transmittance. Low light transmittance is associated with inhibited platelet function, while high light transmittance is associated with normal platelet function. It may be that light transmittance is unchanged in the type of platelet inhibition associated with omega-3 fatty acids. There appears to be conflicting evidence in the cardiac literature as to whether OFA affects platelet function depending on the tests used in the study. ${ }^{17-19}$ Some research has indicated gender specific effects. ${ }^{20}$ There are different hypotheses of the mechanism as to whether OFA affects platelet function, such as through blocking protein kinase signal transduction, ${ }^{21}$ through decreasing CD40-ligand, ${ }^{22}$ or through modulating the fatty acid composition of platelet membranes. ${ }^{23}$ The data in the stroke or cerebrovascular literature in this area is little, if any. OFA may affect platelet function through a different mechanism which cannot be shown by checking P2Y12 inhibition test. It is also unclear whether there is a robust conversion of the OFA to resolvin and protectin molecules, as seen when OFA are used in combination with aspirin. ${ }^{24-26}$ It is these molecules that may inhibit platelet aggregation. There are also other factors which may contribute to OFA effectiveness. It is unclear whether the pathophysiology in the pre-stroke state versus the post-stroke state would alter OFA effectiveness. It is also not clear whether the duration of OFA therapy contributes to greater platelet inhibition. Although not statistically significant, home use, and therefore longer duration of use, prior to hospitalization did result in a higher percentage of patients having adequate P2Y12 platelet inhibition. OFA may not inhibit platelet aggregation directly in that omega-3 fatty acids may have to first incorporate into the cell membranes in order to affect platelet function as previously suggested,27,28 which will take at least 1-2 weeks for one complete platelet to turnover.

One of the limitations of the study is the different time period between the clopidogrel load and the P2Y12 platelet inhibition test. A study had shown that the achievement of sufficiently maximal platelet inhibition with clopidogrel load may not be possible in less than 3 to 4 hours, regardless of the initial loading dose.29 By retrospective nature of this study, the formula of OFA which the subjects were taking at home were different. This could also confound the study as EPA and DHA appear to affect platelet function through different mechanisms and have different potencies. ${ }^{25}$ Patients' compliance could have also confounded the study. Further research is needed to determine if clopidogrel and/or OFA dose and/or duration has an impact on platelet function. Other measures of platelet function, including bleeding time, immune and impedance aggregometry will be useful to measure as part of future studies. Also, aspirin along with OFA use in stroke patients should be investigated as well. Lastly, the long-term outcome of stroke patients on these combined therapies should also be studied to determine if the activity at the cellular and biochemical level is associated with an improved clinical outcome of secondary stroke prevention.

\section{Conclusions}

This study does not completely rule out the possibility of omega-3 fatty acid inhibiting platelet function in stroke patients. However, the concomitant use of omega-3 fatty acid does not appear to improve P2Y12 associated platelet inhibition using the VerifyNow assay.

\section{Strengths and limitations of the study}

I) Omega-3 fatty acids did not provide additional P2Y12 receptor inhibition upon platelets of patients given clopidogrel.

II) Only a single method of inhibition was measured (VerifyNow assay P2Y12 assay).

III) Another mechanism may be involved in platelet inhibition by omega-3 fatty acids

IV) Further study is need to determine if omega-3 fatty acids can have additional platelet function inhibition on patients already on an antiplatelet function medication such as aspirin or clopidogrel.

\section{References}

1. Diener HC, Cunha L, Forbes C, et al.
European stroke prevention study. 2. Dipyridamole and acetylsalicylic acid in the secondary prevention of stroke. J Neurol Sci 1996;143:1-13.

2. CAPRIE Steering Committee. A randomised, blinded, trial of clopidogrel versus aspirin in patients at risk of ischaemic events (CAPRIE). Lancet 1996;348:132939.

3. Furie KL, Kasner SE, Adams RJ, et al. Guidelines for the prevention of stroke in patients with stroke or transient ischemic attack: a guideline for healthcare professionals from the american heart association/american stroke association. Stroke 2011;42:227-76.

4. Amarenco P, Bogousslavsky J, Callahan A 3rd, et al. High-dose atorvastatin after stroke or transient ischemic attack. N Engl J Med 2006;355:549-59.

5. Kramer HJ, Stevens J, Grimminger F, Seeger W. Fish oil fatty acids and human platelets: dose-dependent decrease in dienoic and increase in trienoic thromboxane generation. Biochem Pharmacol 1996;52:1211-7.

6. Deckelbaum RJ, Torrejon C. The omega-3 fatty acid nutritional landscape: health benefits and sources. J Nutr 2012;142: 587S-91S.

7. De Caterina R. n-3 fatty acids in cardiovascular disease. N Engl J Med 2011;364:243950.

8. Savi P, Zachayus JL, Delesque-Touchard N, et al. The active metabolite of Clopidogrel disrupts P2Y12 receptor oligomers and partitions them out of lipid rafts. Proc Natl Acad Sci USA 2006;103:11069-74.

9. Gajos G, Rostoff P, Undas A, Piwowarska W. Effects of polyunsaturated omega-3 fatty acids on responsiveness to dual antiplatelet therapy in patients undergoing percutaneous coronary intervention: the OMEGA-PCI (OMEGA-3 fatty acids after pci to modify responsiveness to dual antiplatelet therapy) study. J Am Coll Cardiol 2010;55:1671-8.

10. Hulot JS, Collet JP, Silvain J, et al. Cardiovascular risk in clopidogrel-treated patients according to cytochrome P450 $2 \mathrm{C} 19 * 2$ loss-of-function allele or proton pump inhibitor coadministration: a systematic meta-analysis. J Am Coll Cardiol 2010;56:134-43.

11. Lee K, Lee SW, Lee JW, et al. The significance of clopidogrel low-responsiveness on stent thrombosis and cardiac death assessed by the verifynow $\mathrm{p}(2) \mathrm{y}(12)$ assay in patients with acute coronary syndrome within 6 months after drug-eluting stent implantation. Korean Circ J 2009;39:512-8.

12. Fox K, Garcia MA, Ardissino D, et al. Guidelines on the management of stable angina pectoris: executive summary: the 
task force on the management of stable angina pectoris of the European Society of Cardiology. Eur Heart J 2006;27:1341-81.

13. Leaf A, Weber PC. Cardiovascular effects of n-3 fatty acids. N Engl J Med 1988;318: 549-57.

14. Kristensen SD, Schmidt EB, Dyerberg J. Dietary supplementation with n-3 polyunsaturated fatty acids and human platelet function: a review with particular emphasis on implications for cardiovascular disease. J Intern Med Suppl 1989;731:141-50.

15. Tilvis RS, Rasi V, Viinikka L, et al. Effects of purified fish oil on platelet lipids and function in diabetic women. Clin Chim Acta 1987;164:315-22.

16. Paniccia R, Antonucci E, Maggini N, et al. Light transmittance aggregometry induced by different concentrations of adenosine diphosphate to monitor clopidogrel therapy: a methodological study. Ther Drug Monit 2011;33:94-8.

17. Serebruany VL, Miller M, Pokov AN, et al. Early impact of prescription Omega-3 fatty acids on platelet biomarkers in patients with coronary artery disease and hypertriglyceridemia. Cardiology 2011;118:18794.

18. von Schacky C, Weber PC. Metabolism and effects on platelet function of the purified eicosapentaenoic and docosahexaenoic acids in humans. J Clin Invest 1985;76: 2446-50.

19. Thorngren M, Gustafson A. Effects of 11week increases in dietary eicosapentaenoic acid on bleeding time, lipids, and platelet aggregation. Lancet 1981;2:1190-3.

20. Phang M, Sinclair AJ, Lincz LF, Garg ML. Gender-specific inhibition of platelet aggregation following omega-3 fatty acid supplementation. Nutr Metab Cardiovasc Dis 2012;22:109-14.

21. McNamara RK, Ostrander M, Abplanalp W, et al. Modulation of phosphoinositide-protein kinase $\mathrm{C}$ signal transduction by omega-3 fatty acids: implications for the pathophysiology and treatment of recurrent neuropsychiatric illness. Prostaglandins Leukot Essent Fatty Acids 2006;75:237-57.

22. Aarsetoy H, Brugger-Andersen T, Hetland 0 , et al. Long term influence of regular intake of high dose n-3 fatty acids on CD40-ligand, pregnancy-associated plasma protein A and matrix metalloproteinase- 9 following acute myocardial infarction. Thromb Haemost 2006;95:32936.

23. Di Stasi D, Bernasconi R, Marchioli R, et al. Early modifications of fatty acid composition in plasma phospholipids, platelets and mononucleates of healthy volunteers after low doses of n-3 polyunsaturated fatty acids. Eur J Clin Pharmacol 2004;60: 183-90.

24. Serhan CN. Resolution phase of inflammation: novel endogenous anti-inflammatory and proresolving lipid mediators and pathways. Annu Rev Immunol 2007;25:101-37.

25. Fredman G, Serhan CN. Specialized proresolving mediator targets for RvE1 and RvD1 in peripheral blood and mechanisms of resolution. Biochem J 2011;437:185-97.

26. Calder PC. Mechanisms of action of (n-3) fatty acids. J Nutr 2012;142:592S-9S.

27. Li Q, Wang M, Tan L, et al. Docosahexaenoic acid changes lipid composition and interleukin-2 receptor signaling in membrane rafts. J Lipid Res 2005;46:1904-13.

28. Ma DW, Seo J, Davidson LA, et al. n-3 PUFA alter caveolae lipid composition and resident protein localization in mouse colon. Faseb J 2004;18:1040-2.

29. Price MJ, Coleman JL, Steinhubl SR, et al. Onset and offset of platelet inhibition after high-dose clopidogrel loading and standard daily therapy measured by a point-ofcare assay in healthy volunteers. Am J Cardiol 2006;98:681-4. 